\title{
Access to inpatient computed tomography pulmonary angiography (CTPA)
}

\begin{abstract}
Introduction : In-patient requests for CTPA should be performed and reported within one working day of receipt and acceptance of the request form, with a target of $90 \%$.
\end{abstract}

Methods: 400 CTPA scans over a three-month period were retrospectively recorded. We noted: Date and time of the request, vetting, scan and report as well as the presence of a PE, Wells score and d-dimer result.

Results and discussion: Scans were reported within 24hours the request in 51.7\% of cases. The target is only reached within 96 hours of the request. The average time from request to report, request to vetting, vetting to scan and scan to report, was 35.3, $11.7,19.39$, and 1.66hours respectively. There was no significant difference for scans requested out ofhours between 17.00 and 09.00 . A PE was diagnosed in $11.3 \%$ of cases. The average time from vetting to scan is the largest portion of the time from request to report. The British Thoracic Society (BTS) guidelines suggest a Wells score $\geq 4$ does not require a d-dimer for which we were non-compliant in $56 \%$ of cases. It also states a CTPA should not be requested without a positive d-dimer if the Well's score is $<4$ for which we were non-compliant in $6.3 \%$ of instances.

\section{Conclusion:}

a. Scrutinise the organisation of the CTPA service.

b. Ensure $100 \%$ compliance with BTS guidelines.

c. Develop an alert system to highlight when the request to report time is approaching 24hours.

d. Develop an electronic CTPA-request form which cannot be submitted unless all clinical information is provided.

Keywords: pulmonary, embolus, CTPA, ct, angiography, d-dimer, wells, score, embolism, PE
Volume 4 Issue 6 - 2017

\section{Muhammad Dharas, Ainkaran \\ Santhirasekaram \\ Department of Radiology, University College London, UK}

Correspondence: Muhammad Dharas, Department of Radiology, University College London, 28 Wyndham Road, Birmingham, UK, Tel 07874995780,

Email mdharas@hotmail.co.uk

Received: November 14, 2017 | Published: November 28, 2017

\begin{abstract}
Abbreviations: CTPA, computed tomography pulmonary angiography; PE, pulmonary embolism; RCR, royal college of radiologists; NICE, national institute for clinical excellence; NHS, national health servicE
\end{abstract}

\section{Introduction}

The RCR advise that the timeframe between receipt and acceptance of an inpatient CTPA and issuing of the report should be one working day, with a recommended target rate of $90 \% .{ }^{1}$ We sought to determine whether this target was achieved within Sandwell and West Birmingham NHS Trust, specifically within the City Hospital site. Our study focuses on the pathway between request and scan, to determine whether the entire process of handling CTPA requests can become more streamlined and thus improve patient care.

A PE occurs when a blood clot, usually a deep vein thrombus from the leg, travels within the bloodstream and lodges within part of the pulmonary arterial network. Although symptoms can be non-specific, a PE commonly results in acute shortness of breath and/or chest pain, but symptoms can also include tachycardia, tachypnoea, haemoptysis, a sense of apprehension and, more rarely, syncope which suggests a larger clot and a poorer prognosis. ${ }^{2}$

Due to the non-specific nature of the symptoms, diagnosing a PE is not always possible clinically. Clinical suspicion relies on history, examination, relevant blood tests including D-dimer where appropriate and when a PE is considered likely, further imaging should be considered to establish a definitive diagnosis. The chance of an abnormal clot forming and subsequently lodging in the pulmonary vasculature is more likely when the components of Virchow's triad are present, ${ }^{3}$ namely; stasis of blood flow, endothelial injury and hypercoagulability. Hence malignancy, significant immobility, major surgery, recent fracture, pregnancy, blood disorders are among the common causes of abnormal clot formation.

It is for this reason that the Wells score - a clinical prediction rule takes into account some of these factors in determining the likelihood of a PE which, together with clinical judgement, point toward or away from further investigations. A CTPA is the gold standard imaging modality in patients who have a clinically likely acute $\mathrm{PE},{ }^{4}$ or a suspected PE with an unlikely Wells score but a positive D-dimer, and according to NICE guidelines it should occur immediately. ${ }^{5}$ In the interim period while awaiting a scan, treatment with an appropriate anticoagulant should begin.

\section{Methods and materials}

We initially analysed retrospective data from 400 in patients who were being investigated for possible PE. This was done using CRIS 
software, chronologically obtaining data from patients who underwent inpatient CTPA across a three month period. The data obtained was: the time of request of CTPA, the time of vetting, the time of scan and the time of report being issued which make up the four main milestones in the scan process, and the requirements outlined by the RCR.

To provide the data with more depth, we also ascertained whether the Wells score was provided as part of the scan request, and based on the Wells score, whether a D dimer was ordered. We obtained this data using CDA software from the hospital intranet. Finally, we read the report in order to prove whether a PE was diagnosed definitively and thus were able to provide an accurate measure of CTPA scans which are actually diagnostic of a PE; in other words, the accuracy of the suspected diagnosis.

To determine whether there was a difference in scans being processed during ordinary workinghours and out ofhours, we separated results obtained from within these timeframes. Workinghours were considered as scans taking place between thehours of 0900-1700, and out ofhours was classified as thehours of 1700-0900.

Following this, the data was subjected to statistical analysis. We initially sought to ascertain lengths of time taken during the reporting pathway, in order to determine timeframes which were promoting or hindering adherence to the RCR target. Additionally, we sought to analyse whether British Thoracic Society guidelines were adhered to especially when requesting a CTPA with a low clinical probability Wells score and associated negative D-dimer, and whether priority was placed on CTPA scans with high clinical probability. We also outlined the timeframe taken for all scans to complete the request to report process, and then separated this data according to Wells score. Finally we ascertained how many pulmonary emboli were actually diagnosed on CTPA, and therefore expressed this as a percentage of the amount of scans ordered.

\section{Results and discussion}

From the data collected from 400 in patients undergoing CTPA, $51.7 \%$ and $68.5 \%$ of scans were reported within 24 hours of the request and vetting respectively, which fell significantly short of the RCR recommended $90 \%$ target. The target was achieved within 72 hours of vetting, at a rate of $92.5 \%$ while $91.3 \%$ of scans were reported with 96hours of the request. It took 192 hours for $100 \%$ of the scans to complete the process from request to report (Figure $1 \&$ Figure 2).

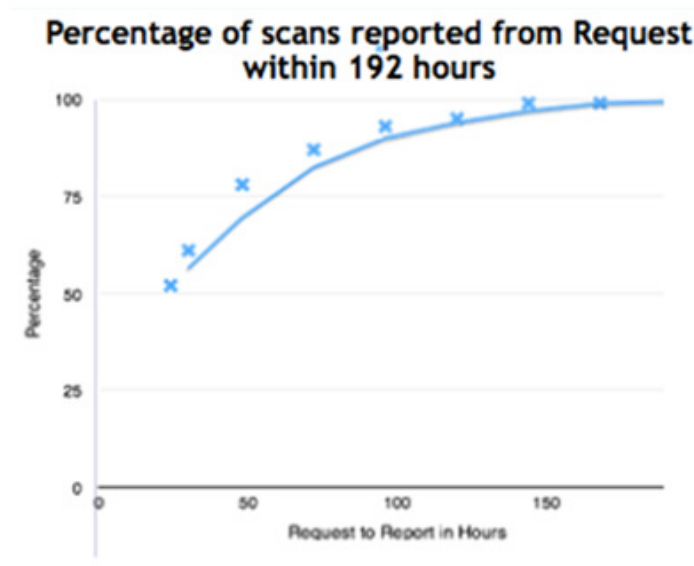

Figure I Percentage of scans reported from request within 192hours.

Average time from request to report, request to vetting, vetting to report, vetting to scan and scan to report, were 35.3 hours, 11.7 hours, 21.04hours, 19.39hours and 1.66hours respectively. For scans requested between 17.00 and 09.00 , average time from request to report, request to vetting, vetting to report, vetting to scan and can to report, was 33.0 hours, 14.1 hours, 18.6 hours, 17.3 hours and 1.68 hours respectively. Request to vetting, vetting to scan and scan to report times are indicated graphically, with working hours compared to out of hours (Figure 3). It demonstrates that the largest proportion of time is spent between vetting to scan (yellow), both during working hours and out of hours.

\section{Percentage of scans reported from Vetting within 192 hours}

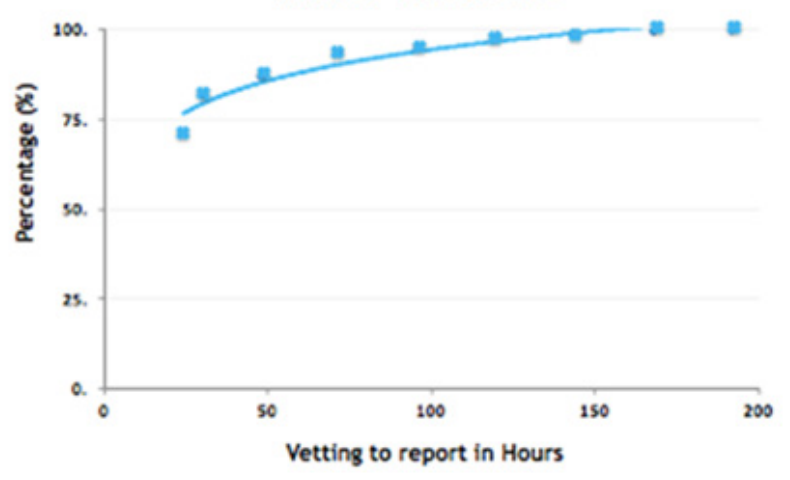

Figure 2 Percentage of scans reported from vetting within 192hours.

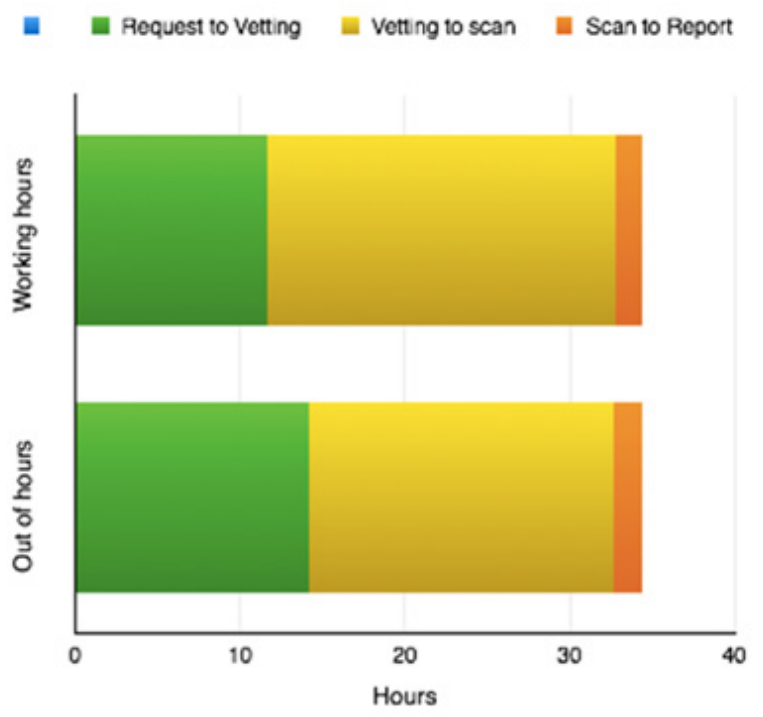

Figure 3 The reporting pathway of working hours vs. out of hours.

Collectively, a PE was diagnosed at a rate of $11.3 \%$. We found a PE present in $15.5 \%$ of cases if the Wells score was greater than or equal to 4 , and in $6.7 \%$ of cases if the Wells score was less than 4 . Importantly, there were no cases of PE when the Wells score was less than 4 and D-dimer was negative. A Wells score was not submitted as part of the request in $4.0 \%$ of cases and a D-dimer was performed in $56 \%$ of cases where the Wells score was greater than or equal to 4; indicating high clinical suspicion of PE. In cases where the Wells score was less than 4 , the D-dimer was negative $1.6 \%$ of the time. Furthermore, the D-dimer was not noted on the request form in $35 \%$ of requests and a D-dimer was not performed in $4.7 \%$ of requests.

The average number of hours from request to report if the Wells score is greater than or equal to 4 and less than 4 was 36.2 hours and 
34.1hours respectively $(\mathrm{p}<0.05)$ (Figure 4). This suggests that there is little discrimination in scanning patients who have a higher clinical probability compared to those with low-medium probability. Based on this, we have designed an easy to use algorithm which will highlight an order of priority when scanning patients with CTPA (Figure 5).

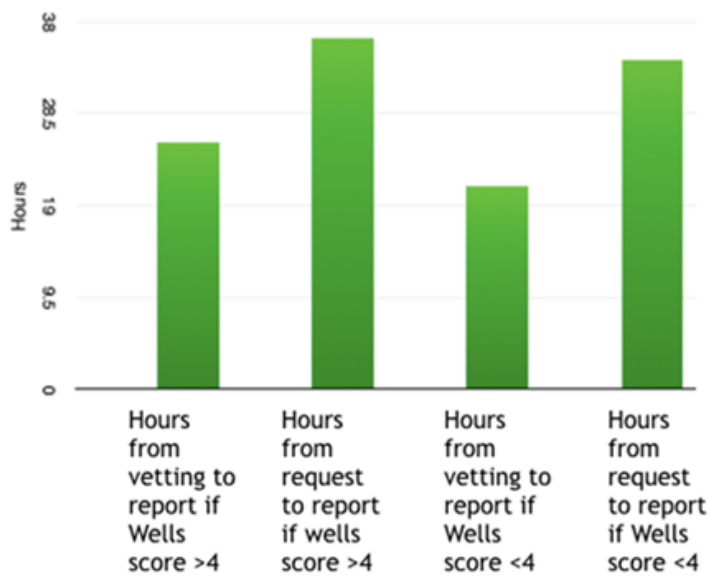

Figure 4 Vetting and request to report times according to wells score.

\begin{tabular}{|c|c|c|c|c|}
\hline $\begin{array}{l}\text { Patients who } \\
\text { a re harm. } \\
\text { dynamically } \\
\text { compromise } \\
\text { d/ ?central } \\
\text { PE }\end{array}$ & $\begin{array}{l}\text { Wells score }>4 \\
\text { and waiting } \\
>20 \text { hours. } \\
\text { Rank based on } \\
\text { time waited } \\
\text { past } 20 \text { hours }\end{array}$ & $\begin{array}{l}\text { Wells score }<4 \\
\text { and waiting } \\
>20 \text { hours. } \\
\text { Rank based on } \\
\text { time waited } \\
\text { past } 20 \text { hours }\end{array}$ & $\begin{array}{l}\text { Wells score }>4 \\
\text { and waiting } \\
<20 \text { hours. } \\
\text { Rank based on } \\
\text { nu m b e r of } \\
\text { hours waited }\end{array}$ & $\begin{array}{l}\text { Wells score }<4 \\
\text { and waiting } \\
<20 \text { hours. } \\
\text { Rank based on } \\
\text { number of } \\
\text { hours waited }\end{array}$ \\
\hline
\end{tabular}

Figure 5 Algorithm highlighting order of priority when using CTPA to investigate patients with suspected PE.

The main outcome of the study was to design an algorithm or pathway which would better enable a Trust to comply with the recommended target outlined by the RCR; for $90 \%$ of scans to be reported within one working day of the request. Our Trust was non compliant by $38.3 \%$, with the target only being achieved within 96hours of the request. As our results demonstrate, the main obstacle in achieving this target appears to be logistical in nature. Although reports are carried out relatively quickly after a scan is performed, obtaining a slot for the physical scan itself seems to take the longest time, and hinders compliance of the target. Furthermore, it seems that implementation of algorithms is suboptimal resulting in overuse of CPTA, often incorrectly.

However, there also appears to be inconsistency in offering CTPA scans. As indicated, $6.3 \%$ of patients with a low probability Wells score and a negative or no D-dimer present still underwent CTPA. In low-medium clinical probability, a D-dimer should be performed because a negative result is helpful in excluding PE in these patients. ${ }^{7}$ Furthermore, when clinical probability is low, offering a D-dimer is part of the most cost-effective strategy in investigating PE. ${ }^{8}$ Generally, D-dimer sensitivity in low probability PE is high, ${ }^{9}$ while one study found sensitivity to be $100 \% .^{10}$

Taking this into account, the number of patients exposed to unnecessary radiation and investigation will reduce, as unquestionably, a high index of clinical suspicion coupled with a likely Wells score result - which pertains to a score of 4 or above, with or without a positive D dimer makes a PE much more likely to be diagnosed on CTPA.
In these cases, performing a D-dimer is not recommended. ${ }^{11}$ However, despite guidelines, we carried out D-dimer tests on $56 \%$ of patients who did not require this i.e. had a high clinical probability. Furthermore, studies have shown that of those investigated for suspected PE, few are confirmed to have the condition. The yield varies from less than $5 \%$ to $30 \%$ based on risk stratification and clinical presentation. ${ }^{12}$ The diagnostic rate of PE in our study was $11.3 \%$ which fits within this range. One possible reason for this is not adhering to BTS guidelines as outlined above. It is safe, within an acceptable failure rate, not scan a patient with a low Wells score and negative D-dimer. ${ }^{13}$ Furthermore, a Wells score of 2 or less can be used alone to reasonably exclude a PE. ${ }^{14}$

Interestingly, a new clinical calculator, known as pulmonary embolism rule out criteria (PERC) has been piloted to determine its efficacy in replacing D-dimer testing. However, despite high negative predictive value, approximately $8 \%$ of confirmed PEs are missed in patients with a low Wells score. ${ }^{15}$

Simple diagnostic algorithms made readily available and displayed in departments such as A\&E have been effective in improving pre-test PE clinical probability documentation, reduced unnecessary D-dimer requests and more importantly reduced the frequency of unnecessary CTPA scans. ${ }^{16}$ In the same way, we hope to implement our algorithm which will not only filter out unnecessary requests but also make prioritizing them a more systematic and streamlined process.

Furthermore, the study as a whole is intended to prove that when organising any radiological intervention, the patient should always be central to the decision. Clinical suspicion should point towards the diagnosis and the appropriate bedside and minimally invasive tests should be carried out in the first instance. This should minimise patients undergoing unnecessary tests which help preserve resources and minimizes exposure to radiation and adverse effects of contrast media. ${ }^{17}$ It is not possible for radiologists to act as gatekeepers to the department, so for the responsible team, acquainting themselves with adequate knowledge of a patient's presentation and background will improve the proportion of appropriate imaging. ${ }^{18}$

\section{Conclusion}

The above algorithm should help to organize and prioritise CTPA requests. However, there is also a logistical requirement of improved efficiency within the department. We recommend that the following improvements will make CTPA requests more likely to impact patient care positively and also help to guide which requests to accept and which to reject, and should also lessen the burden on the department by directing scans in a more organised fashion:

\section{Scrutinise the organisational aspects of the CTPA service}

2. Refuse all CTPA's with a low/moderate two level Wells score and a negative d-dimer or no d-dimer result.

3. Prioritise Well's scores greater than or equal to 4 by advising radiologist to inform radiographers of all patients with a Wells score greater than or equal to 4 .

4. Develop an alert system to highlight radiographers and radiologists when the request to report time is approaching 24 hours.

5. Identifying patients suitable for outpatient pathway.

6. Develop an electronic CTPA-request form which cannot be sub- 
mitted unless all clinical information including well's score and d-dimer are provided.

\section{Ackowledgements}

Dr. Khizer Rana (Consultant Radiologist, Sandwell and West Birmingham NHS Trust).

\section{Conflict of interest}

Author declares that there is no conflict of interest.

\section{References}

1. https://www.rcr.ac.uk/audit/access-lung-scintigraphy-ct-pulmonaryangiography

2. Bělohlávek J, Dytrych V, Linhart A. Pulmonary embolism, part I: Epidemiology, risk factors and risk stratification, pathophysiology, clinical presentation, diagnosis and nonthrombotic pulmonary embolism. Exp Clin Cardiol. 2013;18(2):129-138.

3. Kumar D, Hanlin E, Glurich I, et al. Virchow's Contribution to the Understanding of Thrombosis and Cellular Biology. Clin Med Res. 2010;8(3-4):168-172.

4. Lavorini F, Di Bello V, De Rimini ML, et al. Diagnosis and treatment of pulmonary embolism: a multidisciplinary approach. Multidiscip Respir Med. 2013;8(1):75.

5. https://cks.nice.org.uk/pulmonary-embolism\#! scenario

6. Alhassan S, Abu-Sayf A, Arsene C, et al. Suboptimal implementation of diagnostic algorithms and overuse of computed tomography-pulmonary angiography in patients with suspected pulmonary embolism. Ann Thorac Med. 2016;11(4):254-260.

7. Harringa JB, Bracken RL, Nagle SK, et al. Negative D-dimer testing excludes pulmonary embolism in non-high risk patients in the emergency department. Emerg Radiol. 2017;24(3):273-280.
8. Gerard Stansby. The Management of Venous Thromboembolic Diseases and the Role of Thrombophilia Testing: summary of NICE guidance. The BMJ. 2012;344:e3979.

9. Crawford F, Andras A, Welch K, et al. D-dimer test for excluding the diagnosis of pulmonary embolism. Cochrane Database Syst Rev. 2016;8:CD010864

10. Youssf A, Ismail M, ElGhamry R, et al. Diagnostic accuracy of D-dimer assay in suspected pulmonary embolism patients. Egyptian Journal of Chest Diseases and Tuberculosis. 2014;63(2):411-417.

11. British Thoracic Society guidelines for the management of suspected acute pulmonary embolism. Thorax. 2003;58:470-484.

12. Optimal strategies for the diagnosis of acute pulmonary embolism: a health technology assessment project protocol. Ottawa: CADTH; $C A D$ TH Optimal Use Report. 2016:6(3)

13. Walen S, de Boer E, Edens M, et al. Mandatory adherence to diagnostic protocol increases the yield of CTPA for pulmonary embolism. Insights Imaging. 2016;7(5):727-734.

14. Jeffrey A Kline. Diagnosis and Exclusion of Pulmonary Embolism. Thrombosis Research. 2018;163:207-220.

15. Crane S, Jaconelli T, Eragat M. Retrospective validation of the pulmonary embolism rule-out criteria rule in 'PE unlikely' patients with suspected pulmonary embolism. Eur J Emerg Med. 2018;25(3):185-190.

16. Cooper J. Improving the diagnosis of pulmonary embolism in the emergency department. BMJ Qual Improv Rep. 2015:4(1)

17. Singh J, Daftary A. Iodinated contrast media and their adverse reactions. J Nucl Med Technol. 2008;36:69-74.

18. Bosanquet D, Cho J, Williams $\mathrm{N}$, et al. Requesting radiological investigations - do junior doctors know their patients? A cross-sectional survey. JRSM Short Rep. 2013;4(1):3. 